\title{
Oscillations in Functional Structural Plant Growth Models
}

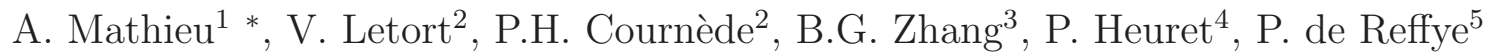 \\ 1 AgroParisTech, UMR EGC (1091), Grignon, France \\ 2 Ecole Centrale Paris, MAS, Châtenay-Malabry, France \\ ${ }^{3}$ China Agricultural University, College of Resources and Environmental Sciences, Beijing, China \\ ${ }^{4}$ INRA, UMR EcoFoG (745), Kourou, French Guyana \\ ${ }^{5}$ CIRAD, UMR AMAP, Montpellier, France
}

\begin{abstract}
The dynamic model of plant growth GreenLab describes plant architecture and functional growth at the level of individual organs. Structural development is controlled by formal grammars and empirical equations compute the amount of biomass produced by the plant, and its partitioning among the growing organs, such as leaves, stems and fruits. The number of organs initiated at each time step depends on the trophic state of the plant, which is evaluated by the ratio of biomass available in plant to the demand of all the organs. The control of the plant organogenesis by this variable induces oscillations in the simulated plant behaviour. The mathematical framework of the GreenLab model allows to compute the conditions for the generation of oscillations and the value of the period according to the set of parameters. Two case-studies are presented, corresponding to emergence of oscillations associated to fructification and branching.

Similar alternating patterns are commonly reported by botanists. In this article, two examples were selected: alternate patterns of fruits in cucumber plants and alternate appearances of branches in Cecropia trees. The model was calibrated from experimental data collected on these plants. It shows that a simple feedback hypothesis of trophic control on plant structure allows the emergence of cyclic patterns corresponding to the observed ones.
\end{abstract}

Keywords and phrases: dynamic system of plant growth, functional-structural plant models, GreenLab, cucumber plant, Cecropia trees

Mathematics Subject Classification: 92B05, 92B15

\section{Introduction}

Plants are complex systems whose dynamics of growth ensures the maintenance and development of vital functions such as exploration and exploitation of its environment to access the resources, transport and conduction of nutriments, mechanical stability and reproduction. To fulfill these multiple objectives, plants produce biomass and invest it in different compartments - stem, roots, leaves, reproductive organs - through processes of growth (stem elongation and thickening), branching, and flowering/fructification.

\footnotetext{
${ }^{*}$ Corresponding author. E-mail: amelie.mathieu@agroparistech.fr
} 
At every growth stage, the expression of these processes is determined by (i) its genotype, (ii) its current structure and functional state and (iii) the amount of available resources (water, light, minerals, gaz). Therefore, different temporal patterns can be identified. For instance, in temperate regions, marked cessation of growth are observed during winter seasons, thus resulting in an annual growth rhythm, with alternation of periods of growth and cessation of growth. These temporal patterns generally affect the plant morphology and anatomy. A well-known example is the presence of growth rings in woody trees, allowing retrospective estimations of their age and providing information about their past conditions (dendrochronology).

Another widely-reported pattern of alternation in tree development is alternate-bearing in fruit crops, widespread throughout many perennial trees and shrubs (apple, pear, mango, beech trees...): a year with intense fruit production is usually followed by a year of poor production [27]. This can be interpreted as a trade-off between allocation to fruits or to reserves [35]. Managing alternate-bearing is a challenge for fruit tree growers; potential remedial measures include intensive horticultural interventions, or introduction of rootstocks or new cultivars [26]. Fluctuations in the dry matter allocation to fruits has also been observed on horticultural plants such as cucumber plants or pepper plants [20]. Fruits are not regularly distributed in the plant, but several phytomers with fruits are followed by phytomers without fruits. Cyclic phenomena are also frequent for tropical trees, although in wet tropical forests, seasonal fluctuations are less marked and environmental cues (temperature, light and rainfall) are not as strong as in temperate zones. Such patterns were reported for two species of the Cecropia genus [12], [37], an emblematic genus of pioneer tree in the neotropics.

However, the main factors that drive the emergence of these temporal or morphological patterns are still poorly known. In particular, characterizing the relative influence of external factors and internal regulations [31], [35] on these patterns is still an open question and is particularly difficult to investigate through observations or experimentations, given the intractable interactions between all these factors and processes.

In that context, the role of modeling is to unravel these complex interactions and to allow testing hypotheses. One key to model these oscillation phenomena lies in the complex interactions between plant architectural development (via organogenesis) and functional growth (via photosynthetic biomass production and allocation) [24]. Mathematical modeling of such phenomena and the analysis of the underlying dynamic system may help underline the conditions for the generation of such oscillations and thus understand the biological processes at their origins.

The objective of this paper is to theoretically and numerically demonstrate the conditions for the emergence of oscillatory patterns in plant architectural growth, and to illustrate the capacity of the model to describe the appearance of such phenomena in real plants. Among the existing functional-structural models [7], the GreenLab model has been chosen to fulfill this aim. Indeed, this model focuses on processes that are generic enough to be applied to most plant species (biomass prodution and allocation, coupled with the plant topological development); its main principles are shared by several other 'carbon-driven' models (e.g. TOMSIM [10], one extension of the ADELwheat model [5] or EcoMeristem [18]) so parts of this work can be generalized; changes in plant topology are dynamically simulated in interaction with the plant physiological state, which allows modelling phenomena such as fruit abortion or branch appearance. Besides, a strong effort was put on its rigourous mathematical formalization, thus paving the way to the study of its behaviour. In GreenLab [3], plant architecture, biomass production and partitioning among organs are described with a discrete dynamic system. The mathematical formalism of the model allows the study of the system behaviour. In a recurrent way, organs are set in place in the plant, biomass is produced by leaves and redistributed among the growing organs. The number of organs initiated at each time step depends on the trophic state of the plant. Hence a vigorous plant can build and support a high number of organs. On the contrary, weak plants will produce fewer organs or even abort some. This model allows testing the potentials and limits of the hypothesis of a development driven by the plant trophic state. It generates oscillatory patterns that can be similar to those observed on real plants [25]. The model considers an average behavior. In real cases, stochastic processes may perturb this behavior, 
while still generating some alternated patterns as shown in [19]. The full study in the stochastic case is still to achieve and the article focuses on the deterministic study.

In Section 2, we first recall the generic concepts of the dynamic system of plant growth used in this paper, and we specify how ecophysiological functioning influences the development of plant organogenesis and structure. We analytically demonstrate that the model generates emergent oscillations of the fructification or branching processes. These theoretical analyses are done for simple versions of the model but they nevertheless provide a sound basis to understand and interpret the properties of more complex versions of the model. In Section 3, we present two illustrations of these results on real plants: alternate fruit bearing in cucumber and alternate branching patterns in Cecropia sciadophylla, a tropical tree. We describe the model equations for these two particular species and their parameter identification from experimental data, and show some numerical simulations of oscillations generated in the structure of these plants. Finally we discuss the potential extensions and applications of this work, in particular for realizing virtual experiments in order to optimize horticultural management strategies.

\section{A Dynamic Plant Model Adapted to the Analysis of Architectural Cyclic Patterns}

\subsection{General Principles of the Model}

\subsubsection{Time and Architecture Discretization}

In this paragraph, we present the generic mathematical frame of the GreenLab model [3, 28]. For applications on real plant species, different versions are derived from this common frame, to adapt to the specificities of each species.

Plant growth equations can be written in the form of a discrete dynamic system. The elementary unit chosen to describe the plant architecture is a botanical entity called phytomer. It is composed of an internode (the part of stem between the insertion points of two successive leaves), one or several leaves (consisting of a blade and potentially of a petiole) and axillary production that can develop into fruits or new branches. When apical or lateral buds blossom, they give birth to new phytomers. A phytomer is modelled as a set of organs. Each organ is characterized by its type $o$ and its chronological age. The organs considered in the model are blades $(o=a)$, petioles $(o=p)$, buds $(o=b)$, internodes $(o=e)$, fruits $(o=f)$. Phytomers are denoted by $m$. All the phytomers of the same chronological age are assumed to be identical. Note that the equations can be easily extended to the case where phytomers have different behaviors but can be classified, according to their morphological properties, in a finite number of classes $[1]$.

The model time step is synchronized with the natural pace of plant architecture setting. It is defined as the time lag between the emergence of two successive phytomers [1].

In the next section, we detail only the equations necessary for our case study but a more comprehensive description can be found in $[3,25]$.

\subsubsection{Model equations}

In GreenLab, the plant structure is modeled by its topology, that is to say the dynamic graph representing the phytomers and their connexions (apical or lateral) [8]. Topological equations thus give the number of new organs at each time step. All the phytomers are assumed to have the same topological properties: they are connected to one apical phytomer at least, and potentially several branches. No branch death nor delays in branch appearance are considered here, but the generalization is straightforward.

If $b(n)$ denotes the number of buds per phytomer at time step $n$ that will give new branches at time step $n+1$, then $v(n)$, the total number of new phytomers at time step $n$, is given by the recurrence equation 2.1. At time step 1 , there is only one phytomer.

$$
\forall n \geq 1, v(n+1)=v(n)(1+b(n))=\prod_{k=1}^{n}(1+b(k))
$$


Functional equations allow to compute biomass production, allocation and organ masses. At time step $n$, the amount of biomass $Q(n)$ produced by the plant is computed by an empirical function $G$ of the plant photosynthetic surface area, called $S(n)$, and of the environmental variables represented in $E(n)$. $P_{f}$ is the vector of the plant functional parameters.

$$
Q(n)=G\left(S(n), E(n), P_{f}\right)
$$

This biomass is shared among the growing compartments of the plant. These compartments can include the growth of newly appeared organs (denoted by index buds) and the expansion of existing organs (index orgs), the constitution of successive layers of rings on axes - for trees - (index rings), the growth of roots (index roots). The parts of the biomass production which are allocated to each of these compartments are determined according to a proportional model [36]: the demand of each compartment is calculated according to specific rules and they share the available biomass proportionally to their respective demands. For instance, the biomass allocated to the expansion of organs is:

$$
\left\{\begin{aligned}
Q_{\text {orgs }}(n) & =D_{\text {orgs }}(n) \cdot \frac{Q(n)}{D(n)} \\
\text { where } D(n) & =D_{\text {buds }}(n)+D_{\text {orgs }}(n)+D_{\text {rings }}(n)+D_{\text {roots }}(n) \\
& =F\left(S(n), Q(n), v(n), N^{o}(1: n), P_{d}\right)
\end{aligned}\right.
$$

$N^{o}(1: n)=\left(N^{o}(1), \cdots, N^{o}(n)\right)$ with $N^{o}(d)$ the number of organs of type $o$ appeared at time step $d$. The total plant demand, $D(n)$, is the demand of the compartments receiving biomass at the beginning of time step $n+1$. It depends on the plant topology, on its current functional state variables and on a set of parameters, denoted $P_{d}$. Then the biomass of each compartment is in turn shared among all its constitutive elements. Let us detail the case of the orgs compartment, which is the core of the dynamic loop of the system. Again, the proportional model is applied to share $Q_{\text {orgs }}$ between all growing organs. The demand of an organ of type $o$ and chronological age $d$ is defined by its sink strength, $p^{o}(d)$ : it measures its ability to attract biomass and is usually modelled by a parametric function. As an organ expands during $T_{e}$ time steps, $p^{o}(d)=0$ when $d>T_{e}$.

$$
D_{\text {orgs }}(n)=\sum_{o \in\{a, p, b, e, f\}} \sum_{d=2}^{\min \left(n+1, T_{e}\right)} v(n-d+1) \cdot N^{o}(n-d+1) \cdot p^{o}(d)
$$

The product $v(n-d+1) \cdot N^{o}(n-d+1)$ represents the number of organs of type $o$ and age $d$. At time step $n$, an organ receives an amount of biomass proportional to the ratio $\frac{Q_{\text {orgs }}(n)}{D_{\text {orgs }}(n)}$ that is equal to $\frac{Q(n)}{D(n)}$ (see equation 2.3).

Regarding the demand of new organs, denoted by $D_{\text {buds }}(n)$, an intermediate variable has to be defined in some cases: if the number of new organs dynamically depends on the current values of some state variables, the demand is first computed taking into account all the potential organs that could appear at the next time step, then the actual number of organs is computed by specific functions, and then the demand value is updated with this information. As an example, the number of new branches $b(n)$ is modeled as a function of the ratio $Q(n) / D(n)$ which is a good measure of the internal competition in the plant and was thus chosen as a control variable for plant self-regulation [24]:

$$
b(n)=H\left(Q(n) / D(n), P_{t}\right)
$$

with $P_{t}$ the plant topological parameters.

The plant photosynthetic surface area is the sum of the surface areas of all the photosynthetic organs. In this article, only leaf blades are supposed to be photosynthetic and they remain active while their chronological age is lower than $T_{a}$. The surface area of one leaf blade is the ratio of its mass divided by 
its mass per unit area, which is supposed constant for all leaves and denoted by $e$. Equation 2.6 therefore gives the general equation to compute the photosynthetic surface area.

$$
S(n)=\frac{1}{e} \sum_{d=1}^{T_{a}} v(n-d+1) \cdot N^{a}(n-d+1) \sum_{k=1}^{d} p^{a}(k) \frac{Q(n-d+k-1)}{D(n-d+k-1)}
$$

Equations 2.1 to 2.5 define the reccurence equations of plant growth and the plant growth model thus comes in the form of a discrete dynamic system.

\subsubsection{Model Parameterization}

Apart from the parameters that can be measured directly on plants, some ecophysiological parameters of the functional equations (parameters $P_{f}$ from Equation (2.2) and parameters of the empirical sink functions $p^{o}$ ) and some topological parameters $\left(P_{t}\right.$ from Equation $\left.(2.5)\right)$ need to be estimated from experimental data by model inversion, as detailed by [4]. If plant topology is given (that is to say if the development sequence $v(n)$ is known and $P_{t}=\emptyset$ ), the model outputs are continuous functions of the parameters and continuous optimization methods can be applied straightforwardly for likelihood maximization or generalized least squares minimization. Regarding topological parameters driving the retroaction of functioning on organogenesis $P_{t}$, Equation (2.5) generally includes transformations from real values to integers. Hence, it does not generate continuous variations of the model outputs and Newton type methods can no longer be applied. We need to resort to heuristic methods to minimize the cost function. We choose the simulated annealing algorithm [14].

Owing to the relative simplicity of functional-structural interactions in the GreenLab model, it is possible to perform the estimation of topological and ecophysiological parameters in a recursive loop with separated steps. In a first step, the topology is fixed to the measured one (that is to say that sequences $b(n)$ and $v(n)$ are considered given). It allows the estimation of the ecophysiological parameters with a continuous method. Once these functional parameters are estimated, topological data (e.g. number of branches or organs) are added into the target observation data and the topological parameters are estimated while ecophysiological data are considered known. Several trials are usually needed to ensure convergence to a reasonable solution with the simulated annealing.

Theoretical analyses of the model properties in terms of emergent oscillatory patterns have been carried out on two case studies: fructification and branching.

\subsection{Theoretical analysis of the generation of oscillations for fructification and branching processes}

Based on the generic model frame described above, we derive the equations of two models: one is designed to analyze the fructification process and the other one the branching process. In both cases, we analyze the theoretical conditions for the emergence of oscillations. Previous works on such mathematical studies can be found in $[22,25]$. These two simplified case-studies correspond to observations that have been widely reported by botanists but that are not well understood from a biological point of view. Mathematical studies focus on the sequence $\left(\frac{Q(n)}{D(n)}\right)_{n \in \mathbb{N}}$ regarding the important role played by this variable in the model equations.

\subsubsection{Conditions for the Generation of Oscillations associated to Fructification}

Let us consider a single-stem plant, with one new phytomer appearing at each time step. In that case, phytomers are composed of one internode, one leaf and one flower. The flower may or not become a fruit depending on the plant trophic conditions. In the model, we assume that the flower aborts if the ratio of biomass to demand is below a given threshold. Only the orgs compartment is considered.

In this case-study, the empirical function $G$ (Equation 2.2) is based on the Beer-Lambert approximation, where $P_{f}=\left\{\mu, S_{p}, k\right\}$ are crop parameters. Leaves are supposed to be photosynthetic during $T_{a}$ time 


\section{TABLE 1. Notation}

\begin{tabular}{cc}
\hline Name & Description \\
\hline$Q(n)$ & Biomass produced at time step $n$ \\
$D(n)$ & Plant demand at time step $n$ \\
$d^{r}(n)$ & Demand of the ring compartment \\
$D_{v}$ & Demand of vegetative organs $\left(=\left(p^{a}+p^{e}\left(T_{e}\right)\right)\right.$ \\
$N^{o}(n)$ & Number of organs of type $o$ appeared at time step $n$ \\
$N^{f}(n)$ & Number of infructescences on a growth unit appeared at $n$ \\
$b(n)$ & Number of buds per phytomer at time step $n$ \\
$v(n)$ & Number of new phytomers at time step $n$ \\
$E$ & Environment input variable \\
$S(n)$ & Total blade surface \\
$\mu, S_{p}, k, R$ & Crop parameters involved in functional equations \\
$e$ & Leaf thickness \\
$T_{a}$ & Number of time steps for a photosynthetic leaf \\
$T_{e}$ & Number of time steps for organ to grow \\
$p^{o}(d)$ & Sink strength of organ of type $o$ and chronological age $d$ \\
$p_{k}^{b}$ & (replaced by $p^{o}$ if sink strength is independent on $\left.d\right)$ \\
$o$ & Sink stength of a new phytomer of rank $k$ (cecropia) \\
$B$ & Type of organ (a: blade, e: internode, f:fruit, p:petiol) \\
$A$ & $\frac{k p^{a}}{S_{p} e}$ \\
$b$ & $E \mu$ \\
$C$ & $B \frac{T_{e}\left(T_{e}+1\right)}{2}$ \\
$\theta$ & $\frac{e\left(p^{e}+p^{a}\right)}{\mu p^{a}}$ \\
$m$ & Threshold for fruit apperance \\
$\lfloor x\rfloor$ & Number of leaves per phytomer part of $x$ \\
\hline
\end{tabular}

steps, i.e. during their growth period. Hence the amount of biomass produced at time step $n$ is given by the following relationship:

$$
Q(n)=E \mu\left(1-e^{-k \frac{S(n)}{S_{p}}}\right)
$$

As the other compartments are not considered here, the plant demand $D(n)$ is the organ demand $D_{\text {orgs }}(n)$. There is only one new organ of each type (leaf, internode, fruit) at each time step, and organs are growing during $T_{e}$ time steps. It is assumed that the organ sink variation does not depend on the organ age unlike the real plant parametric function represented in 4 . Hence equation 2.4 is equivalent to the following equation:

$$
D(n)=\min \left(n+1, T_{e}\right)\left(p^{e}+p^{a}\right)+\sum_{d=1}^{\min \left(n+1, T_{e}\right)} p^{f}(d) \delta(n-d+1)
$$

$\delta(n)$ denotes a variable equal to 0 if the flower created at time step $n$ aborts and 1 if the corresponding fruit grows. In the model, a fruit grows on the phytomer appeared at time step $n$ if $\frac{Q(n+d)}{D(n+d)}>\theta$, with $\{d, \theta\} \in P_{t}$. There is a delay between the fruit initiation and the beginning of its growth.

Let us denote $B=\frac{k p^{a}}{S_{p} e}, A=E \mu, D_{v}=\left(p^{a}+p^{e}\right) T_{e}, b=B \frac{T_{e}\left(T_{e}+1\right)}{2}$. We define the sequence $\left(\alpha_{n}=\right.$ 
$\left.\frac{Q(n)}{D(n)}\right)_{n \in \mathbb{N}}$. From equations $2.6,2.7,3.1$, this sequence $\alpha$ is defined by the recurrence equation:

$$
\alpha_{n+1}= \begin{cases}A \frac{1-e^{-B \sum_{d=1}^{n} d \alpha_{d}}}{\left(p_{e}+p_{a}\right)(n+1)+p^{f} \sum_{d=1}^{n+1} \delta\left(\alpha_{n+1-d}\right)} & \text { if } n<T_{e}, \\ A \frac{1-e^{T_{e}} \sum_{d=1} d \alpha_{n-T_{e}+d}}{D_{v}+p^{f} \sum_{d=1}^{T_{e}} \delta\left(\alpha_{n+1-d}\right)} & \text { if } n \geq T_{e},\end{cases}
$$

Three behaviours are observed according to parameter values:

1. If $A b<D_{v}$, the sequence $\alpha$ tends to 0 , the plant cannot bear any fruit (see appendix 4).

2. If $D_{v}<A b<D_{v}+T_{e} p^{f}$, the simulations present alternating patterns of fruits on the main stem, i.e. sequences of phytomers without fruits followed by sequences of phytomers bearing fruits.

3. If $A b>D_{v}+T_{e} p^{f}$, there is a fixed point for the function $f: x \rightarrow f(x)=\frac{A}{D_{v}+T_{e} p^{f}}\left(1-e^{-b x}\right)$. If this fixed point is greater than the threshold, all the fruits can grow. Otherwise, alternating patterns of fruits emerge on the stem.

These results are illustrated by numerical simulations of these three behaviours (fig. 1). The plant parameters $\left(P_{f}, P_{t}\right)$ are identical in the three examples, computed from model calibration (see section 3.1.2) but sink strengths are supposed constant all along organ expansion. The threshold value for fruit growth is $\theta=0.5$. The model is tested with three levels for the environment input variable $E$, which give three values for the parameter $A$ corresponding to the three cases presented above. When $E=0.15$, $A b<D_{v}$, hence the ratio of biomass to demand tends to 0 and so do the phytomer masses. No fruit is produced by the plant. When $E=1, D_{v}<A b<D_{v}+T_{e} p^{f}$, alternating patterns are produced, as the ratio of biomass to demand sometimes exceeds $\theta$, which induces fruit appearances and consequently an increase in plant demand. This leads to the decrease of the ratio which becomes lower than $\theta$. It induces flower abortion and so on. When $E=2, A b>D_{v}+T_{e} p^{f}$, the sequences converges to the value 0.5844 which is above the threshold. All the flowers will become fruits.

\subsubsection{Branching}

In this section, we consider a tree whose type corresponds to the Leeuwenberg architectural model [13]. Phytomers consist of an internode and $m$ leaves; they bear $m$ axillary buds, each of them can potentially give rise to a new lateral branch. In the case of Leeuwenberg architectural models, the apical bud die and is relayed by the axillary ones. It is the case considered in this study but this can be easily extended to trees for which apical buds stay alive. No bud dormancy is considered: buds either produce a branch at the time step after their initiation, or die. Bud outgrow is assumed to depend on the tree state of internal trophic competition, represented by the ratio of available biomass to demand, $Q / D$ : the number of new phytomers at time step $n$ is given by equation 2.5, with $H$ a step function. $b(n)$, the number of buds per phytomer at time step $n$ that will give new branches at time step $n+1$, is given by the following equation:

$$
b(n)=\left\lfloor\frac{1}{\theta} \frac{Q(n)}{D_{t e m p}(n)}\right\rfloor
$$

where $\lfloor x\rfloor$ denotes the integer part of $x$. $\theta$ denotes the threshold value to be exceeded to produce an additional branch. In this equation, $D_{\text {temp }}(n)=D_{\text {orgs }}(n)+D_{\text {rings }}(n)+D_{\text {roots }}(n)+D_{\text {buds }}(n)$. $D_{\text {temp }}(n)$ is the demand of equation 2.3 with the potential number of new branches, including the sinks of the potential buds. It is used to compute the number of new branches that can emerge at the current cycle, and it is then updated to $D(n)$, the effective demand, once the number of new organs is computed. 

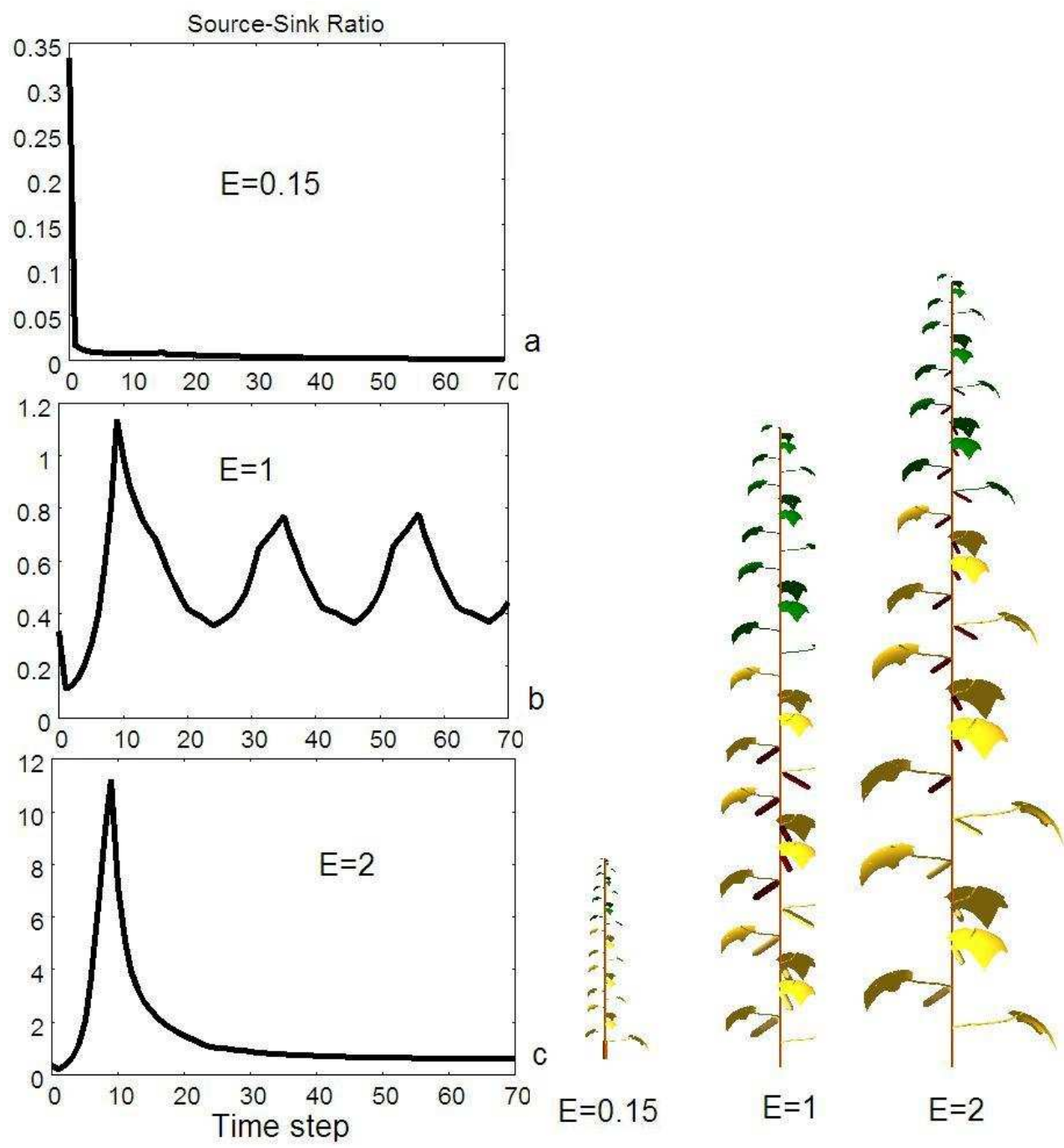

$E=1 \quad E=2$

FiguRE 1. Simulation of the $Q / D$ ratio with three different values for the environment variable $E$ and corresponding plant representations. a. $E=0.15$ the sequence tends to 0 and all the flowers abort. b. $E=1$ Cyclic patterns of fruits are observed on main stem. c. $E=2$. The sequence $\alpha$ tends to a value that is above the threshold for fruit growth, hence all the flowers become fruits.

The production function $G$ is assumed to be an hyperbolic function of blade area, $S(n)$. Equation 2.2 becomes:

$$
Q(n)=\frac{E}{\frac{1}{\mu \cdot S(n)}+R}
$$

As for equation 2.7, $\mu$ and $R$ are parameters related to the biomass conversion efficiency and $E$ is linked to the environmental conditions. Here again, only the orgs compartment is considered and we assume that organs reach their final sizes immediately $\left(T_{e}=1, T_{a}=1\right)$. Let us denote $C=\frac{e\left(p^{e}+p^{a}\right)}{\mu p^{a}}$. From [22], we know that 
- if $(E-C)>R \cdot m \cdot \theta, m$ branches appear at each time step. The number of leaves and the biomass production increase in an exponential way.

- if $\exists k \in\{0, \ldots, m-1\}$, such that $R \cdot m \cdot \theta k<E m-C k<R \cdot m \cdot \theta(k+1)$, then $k$ out of $m$ new branches appear at each time step.

- otherwise, alternating patterns appear in the branching system: the number of new branches oscillates between $k$ and $k+1$ such that $E m-C(k+1)<R(k+1) \theta m<E m-C k$.

When alternating patterns emerge, it is possible to give an integer approximation of the period:

$$
\hat{p}=1+\frac{\ln \left(\frac{E m-C k-R m(k+1) \theta}{m \theta(k+1)\left(\frac{C(k+1)}{m \theta(k+1)}\left(1-\frac{C k}{E m}\right)-B \frac{C k}{E m}\right)}\right)}{\ln \left(\frac{C k}{E m}\right)}
$$

These behaviours are illustrated on figure 2. The period is not an integer with this set of parameters. The number of new branches oscillates between 1 and 2 per phytomers, with the alternating pattern $: 2-1-2-1-1-2-1$. The trend of the ratio of biomass to demand highlights this periodic pattern.
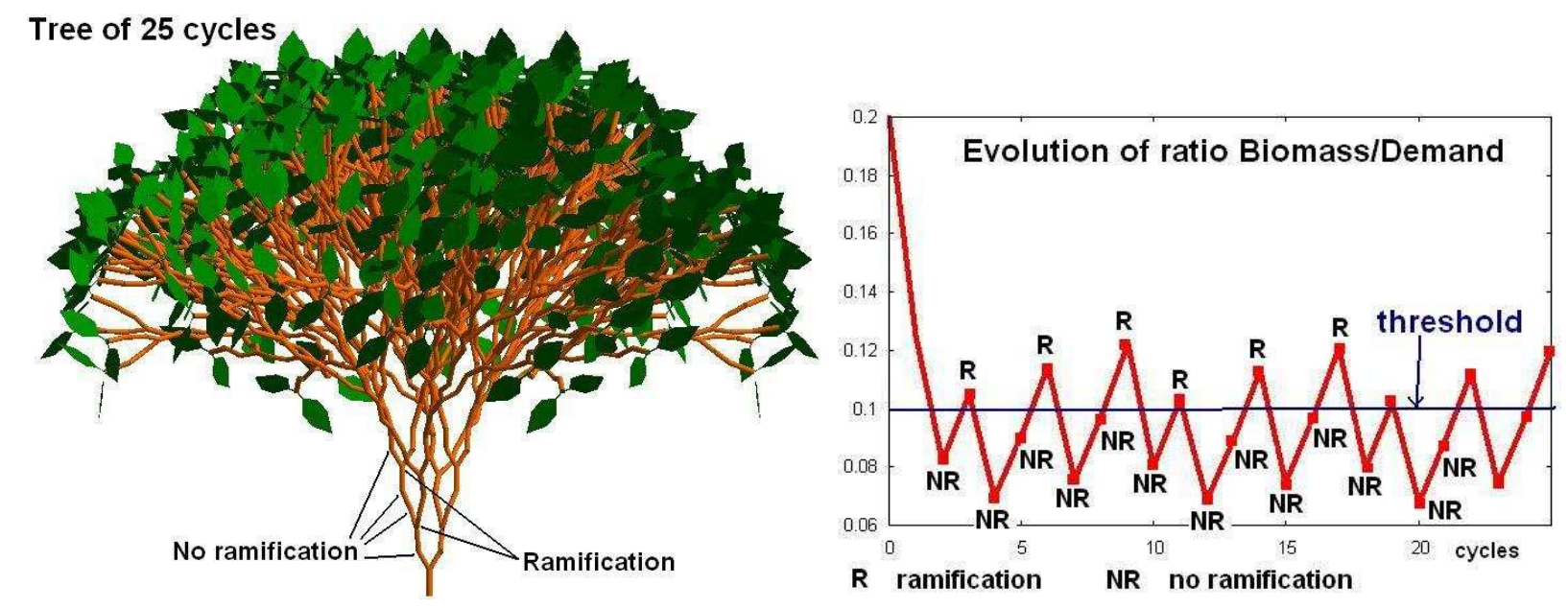

FIgURE 2. Simulation of plant growth and corresponding trend on the ratio of biomass to demand. Parameter values are $: R=1, m=2, C=1.4$. Thus, $\hat{p}=2.57$. $N R$ and $R$ respectively stand for 'No Ramification' and 'Ramification'.

\section{Application to real plants and simulations}

\subsection{Cucumber Plants}

In this section, an application of the model to cucumber plants is presented. A few specific adaptations are implemented in the model for application to the data without changing the main hypotheses of the model.

\subsubsection{Model Equations for Cucumber}

Unbranched plants are considered, which corresponds to the horticultural practice of branch pruning. Hence $\forall n, v(n)=1$. The growth of pruned Cucumber plants thus corresponds to the case-study presented 
in section 2.2.1 with addition of petioles in the model and without the hypothesis of constant sinks. The resulting equation of the demand is therefore adapted from equation 2.8 , with the same notations, as follows:

$$
D(n)=\sum_{d=1}^{\min \left(n+1, T_{e}\right)}\left(p^{e}(d)+p^{a}(d)+p^{p}(d)\right)+\sum_{d=1}^{\min \left(n+1, T_{e}\right)} p^{f}(d) \delta(n-d+1)
$$

If $n \geq T_{e}, D_{v}=\sum_{d=1}^{T_{e}}\left(p^{e}(d)+p^{a}(d)+p^{p}(d)\right)$ is independent on $n$ and the only varying factor in the computation of the demand is the number of growing fruits.

The sequence $\left(\alpha_{n}=\frac{Q(n)}{D(n)}\right)_{n \in \mathbb{N}}$ follows the recurrent equation when $n \geq T_{e}$ :

$$
\alpha_{n+1}=E \mu \frac{1-\exp ^{-\frac{k}{e S_{p}} \sum_{d=1}^{T_{e}} \sum_{k=1}^{d} p^{a}(k) \alpha_{n-d+k}}}{D_{v}+\sum_{d=1}^{T_{e}} p^{f}(d) \delta(n+2-d)}
$$

(to be compared with equation 2.9).

\subsubsection{Model Calibration}

Cucumber plants (Cucumis sativus cv. JingYan Mini2 from the Beijing Academy of Agricultural and Forestry Science) were grown in a greenhouse of Beijing Academy of Agricultural Sciences (BAAS) from March 20th to June 15th 2006. Only natural daylight was supplied for plant growth. Air temperature inside the greenhouse varied from $15^{\circ} \mathrm{C}$ to $30^{\circ} \mathrm{C}$. Individual seedlings with one true leaf were planted in $29 \times 24 \times 25 \mathrm{~cm}$ containers, containing $1: 2(v / v)$ vermiculite and peat mixed with $0.25 \mathrm{~kg}$ of poultry compost. The containers were arranged in rows with row spacing of $1 \mathrm{~m}$ and $0.5 \mathrm{~m}$ within a row. The plot size was $100 \mathrm{~m}^{2}$. Measured plants were pruned by removing all axillary buds immediately after appearance. Four plants were randomly harvested at three different dates (May 10, May 17 and June 13). They were separated into root system and shoot, with the shoot further divided into phytomers. Each organ was dry weighted and the leaf surface areas were estimated by analysis of leaf photographs. Although model calibration has been carried out on several measured plants, it is only presented here for one plant (figure 3), the other results being similar. The growth model was calibrated on plants measured at the three dates, as if it were the same plant growing, which is not possible due to destructive measurements. For each plant at the final stage, youngest plants were selected according to their number of phytomers.

Model calibration allows us to reproduce the growth of the observed cucumber plants in terms of growth rate and partitioning. Some parameters are directly deduced from measurements, such as $T_{e}$ and $T_{a}$ which are set to 20 time steps and $e=0.02 \mathrm{~g} \cdot \mathrm{m}^{-2} . E=8.8 \mathrm{MJ}$ is proportional to the photosynthetically active radiation in the greenhouse during one time step. The other parameters are estimated using the method described in section 2.1.3. For equation 2.7, $S_{p}=0.44 \mathrm{~m}^{2}$, and $\mu=95.8$. Estimated topological parameters are $\theta=0.5, d=7$. The parametric functions for organ sink strength and variation (parameters $\left.p^{o}(d), d \in\left\{1, T_{e}\right\}\right)$ are represented on figure 4 .

There is a satisfying adequacy between measurements and model simulation for the masses of organs. The model simulates more growing fruits than really observed on the measured plant (Figure 3D). The ratio of well predicted position to the total potential positions for fruits is $89 \%$.

\subsubsection{Cyclic Patterns of Fructification in the Calibrated Model}

The estimation procedure allows deducing the pattern of the source-sink ratio (Figure 5) from the plant architecture. This ratio exceeds the threshold parameter twice in the plant growth which induces two waves of fruit on the stem. When the curve is above the horizontal line (threshold), fruits grow. Otherwise they abort. This ratio present a cyclic pattern. Indeed, when the ratio is high enough, initiated fruits can grow which induces an increase in plant demand and then a decrease of the ratio of biomass to 

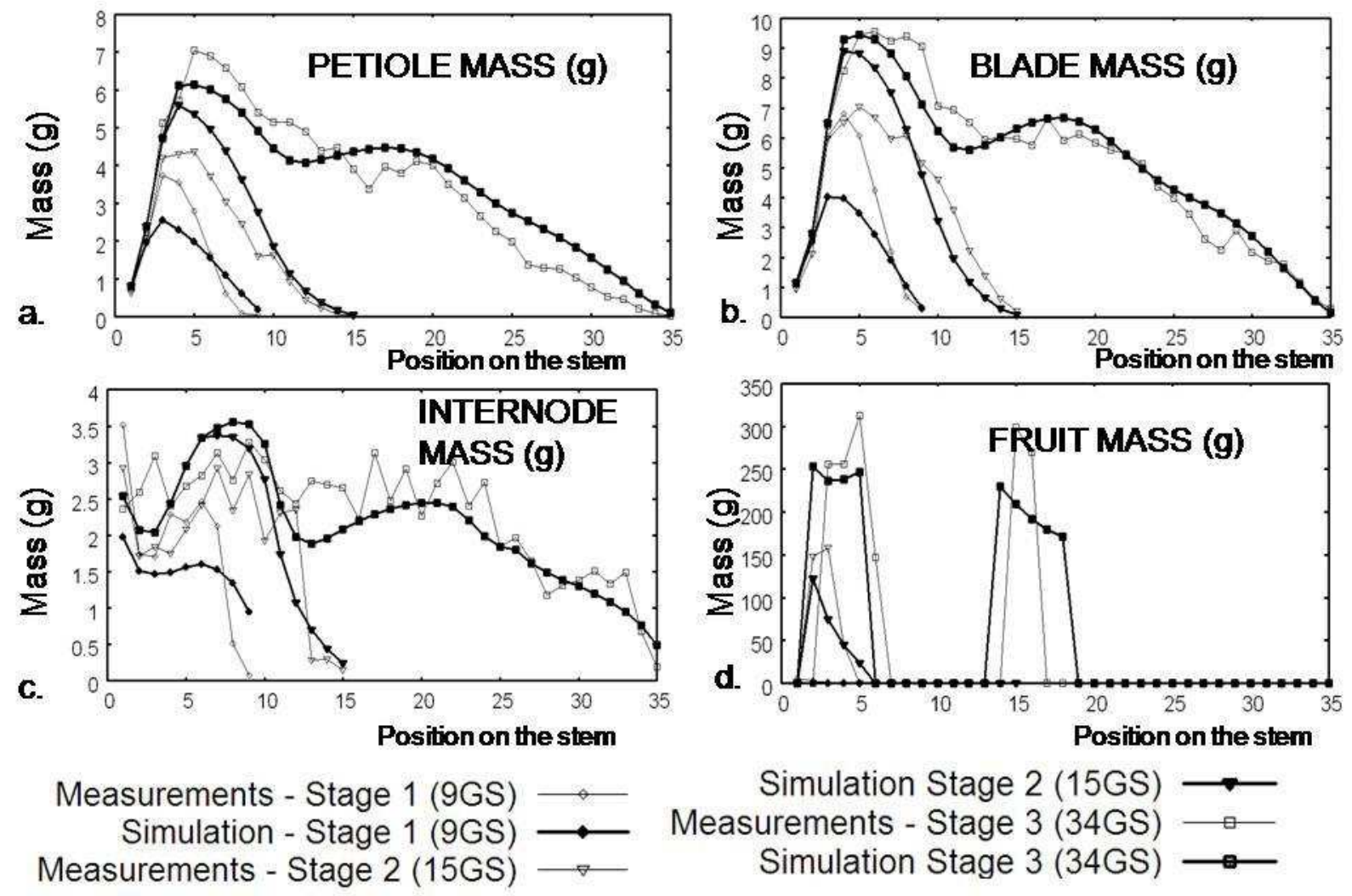

Figure 3. Model calibration on 4 output variables according to the phytomer rank on the main stem A. Petiole Mass. B. Blade Mass. C. Internode Mass. D. Fruit Mass

demand. This ratio goes behind the threshold so fruits abort again, inducing a decrease in plant demand and so on... Along the stem, this balance between biomass production and demand results in alternating succession of fruit and fruit-free phytomers. It also impacts the leaf profile (figure 6). Leaves on the fruit-bearing phytomers compete with fruits for biomass and hence have smaller leaf area comparing to leaves growing without fruit competition. Section 2.2 theoretically explains how oscillations are generated in the dynamic system of plant growth.

\subsection{Cecropia trees}

Cecropia sciadophylla Mart. is a tropical tree species that has a distribution area covering the Amazonian basin, the Llanos region in Columbia and Venezuela, and the Guiana shield [2]. Trees of this species have interesting properties from a modeler's point of view: in particular, contrary to what is experimentally feasible for most tree species, exhaustive descriptions of the plant structure and mass partitioning are accessible. C. sciadophylla trees grow rapidly and continuously: one phytomer is emitted every two weeks on the main stem. They have a simple architecture made of a relatively low number of phytomers, although their life span can reach 40 years (for example, an eleven-year-old measured individual of $C$. sciadophylla was constituted by approximately 1700 phytomers). The succession of internodes and their associated lateral production remain visible over years (Figure 7). Each node bears three lateral buds 

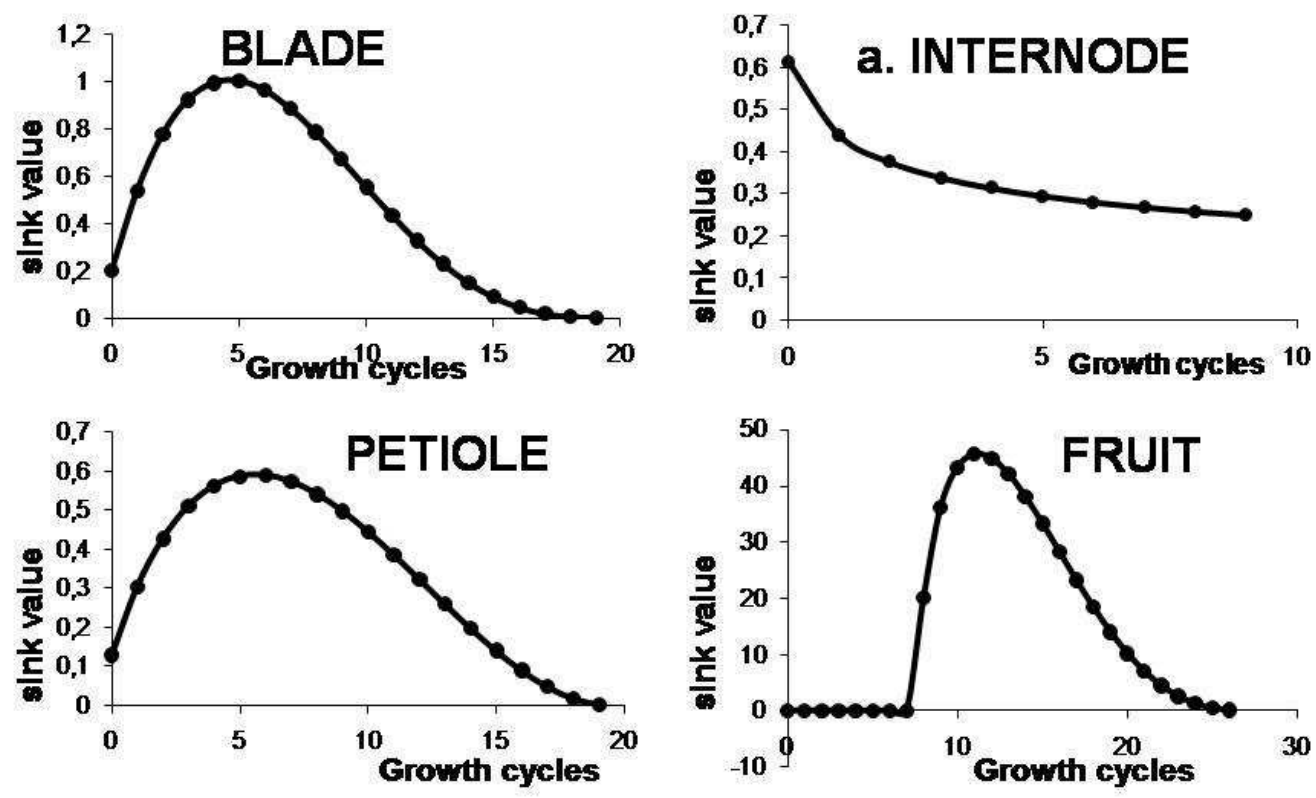

FIGURE 4. Sink strength variations with time step for blades, internodes, petioles and fruits after estimation from experimental data.

that potentially give rise to a branch (central bud) and two infructescences [37], that are alternatively activated: synchronous branching periods cyclically alternate with flowering periods $[12,37]$. The origins of these periodic patterns is questioned in [16]: it was shown that, among other factors, dynamic trophic competition during the plant development could be involved.

In this section, we present the equations of the model for C. sciadophylla and its calibration from experimental data.

\subsubsection{Model Equations}

The plant topology consists in a sequence of phytomers along the trunk, that can potentially bear two infructescences and one branch. Infructescence positions are fixed from the observations. Branches are supposed to appear if and only if the ratio of biomass to demand is above a threshold denoted by $\theta$, as in Equation 2.10. Biomass production is assumed to be proportional to photosynthetic surface area, as self-shading can be considered as negligible for Cecropia due to quasi-optimal arrangement of leaves [15], [16]. Hence the production function $G$ is linear as in the case-study of section 2.2.2 (equation 2.11 with $R=0$ ). In contrast with the case-study 2.2 .2 , roots and rings compartments have to be considered for real applications on trees. 


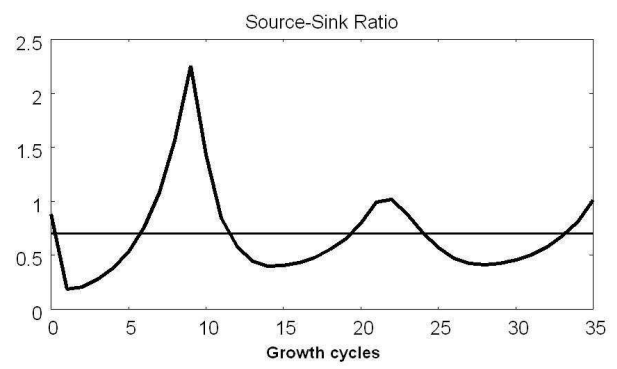

Figure 5. Trend of the ratio of biomass to demand with time

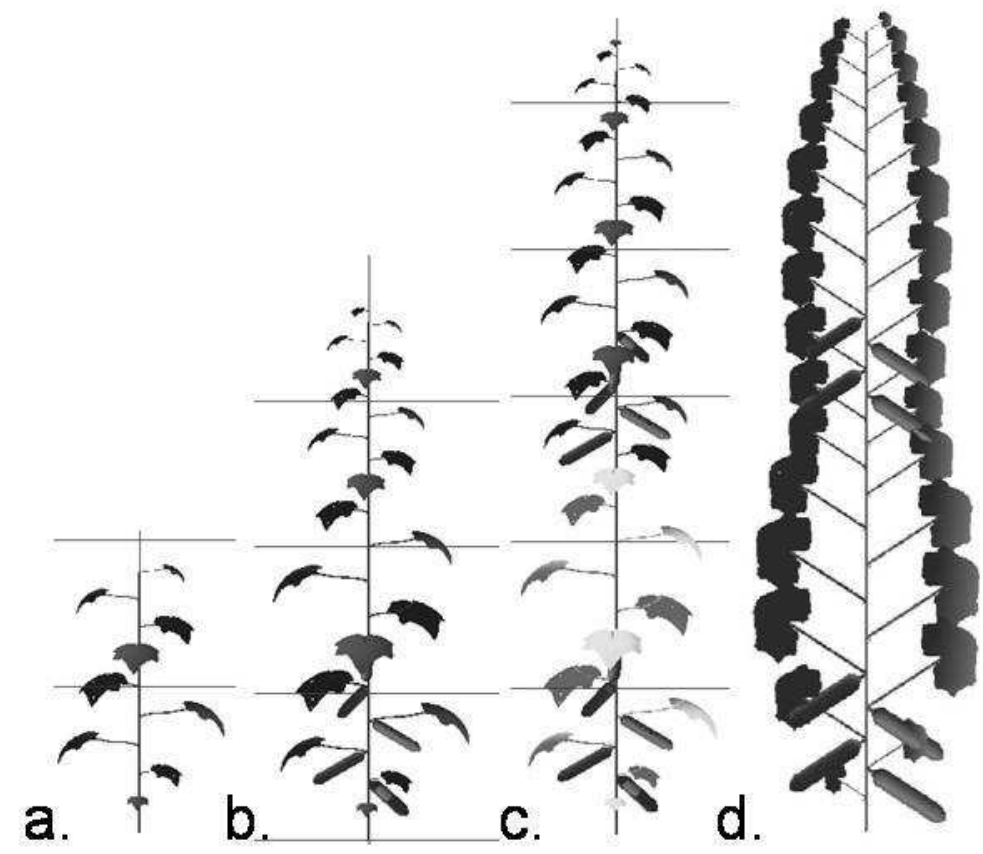

Figure 6. Visualization of the simulated cucumber plant at time steps 9 (a), 20 (b), 31 (c). The final plant is represented in $2 \mathrm{D}$ to show the cyclic leaf profile. Dark leaves are still photosynthetically active, pale leaves are dead.

The demand of the plant at time step $n>0$ is thus:

$$
\begin{array}{r}
D_{\text {temp }}(n)=p_{1}^{b} \cdot v(n)+\sum_{k=2}^{n} p_{k}^{b} \cdot b(n-k+1) \cdot v(n-k+1) \\
+\sum_{d=1}^{T_{e}} p^{f}(d) \cdot N^{f}(n-d+1) \cdot v(n-d+1)+d^{r}(n)
\end{array}
$$

We remind that $N^{f}(n)$ is the number of infructescences on a growth unit that appeared at time step $n$ and that $v(n) \cdot b(n)$ is the total number of buds that will give new branches at time step $n+1$. Since it is assumed that no axis dies and that there is no bud dormancy, the apical phytomer of each branch (including the main stem) bear two potential buds: the apical one, of rank $k$, and the lateral one, of rank 1 ( $k=1$ at the axis basis). The sink $p_{k}^{b}$ of a new phytomer varies with its rank $k$ along an axis to account 


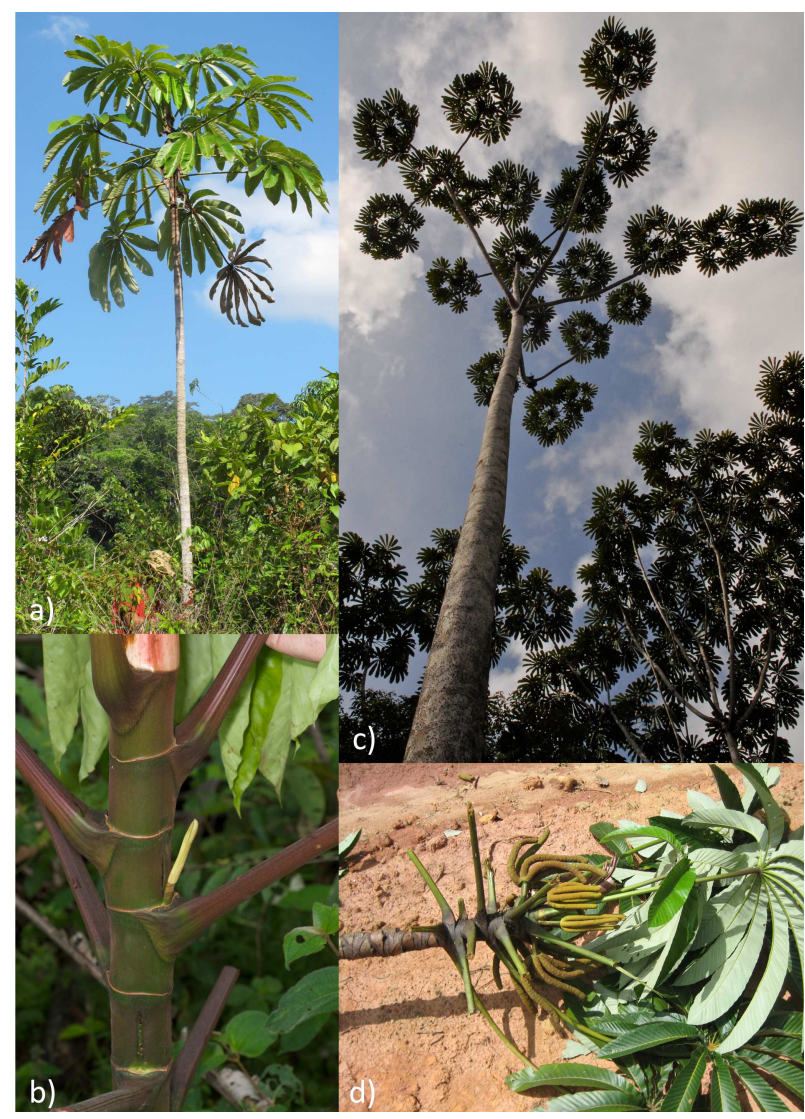

Figure 7. Habit of C. Sciadophylla: (a): young individual, (b): appearance of a young branch, (c): crown architecture of an adult individual, (d): infructescences on an adult individual.

for the ontogenetical variations during the establishment phase at branch emergence [16]:

$$
p_{k}^{b}=p^{b} \cdot \min \left\{1, k V^{b}\right\}
$$

where $p^{b}$ is the bud sink in the stable phase of branch growth and $V^{b}$ is the slope parameter.

The demand for the ring compartment at time step $n, d^{r}(n)$, is assumed to depend on the total blade surface $S(n)$, in reference to the widely used pipe model theory [32]:

$$
d^{r}(n)=p^{r} \cdot S(n)
$$

where $p^{r}$ is the sink strength of the ring compartment. Once the potential demand $D_{p b}$ is calculated, the number of new branches per phytomer at time step $n+1$, corresponding to $b(n)$, is computed according to equation 2.10 and the effective demand can be calculated as:

$$
\begin{array}{r}
D(n)=\sum_{k=1}^{n} p_{k}^{b} \cdot b(n-k+1) \cdot v(n-k+1) \\
+\sum_{d=1}^{T_{e}} p^{f}(d) \cdot N^{f}(n-d+1) \cdot v(n-d+1)+p^{r} \cdot S(n)
\end{array}
$$

Note that the first term of the demand includes only the demand of buds that will outgrow: indeed, if the threshold condition of equation 2.10 was not satisfied, $b(n)=0$. We assumed that only lateral buds 
TABLE 2. Values of estimated functional parameters

\begin{tabular}{lccccccccccc}
\hline Env. Factor & $E^{1}$ & $E^{2}$ & $E^{3}$ & $E^{4}$ & $E^{5}$ & $E^{6}$ & $E^{7}$ & $E^{8}$ & $E^{9}$ & $E^{10}$ & $E^{11}$ \\
& 7.7 & 7.5 & 7.3 & 5.7 & 3.3 & 6.5 & 6.4 & 6.3 & 15.9 & 10 & 7.2 \\
\hline Funct. Params & \multicolumn{1}{c}{$p^{r}$} & \multicolumn{2}{c}{$p^{f}$} & \multicolumn{1}{c}{$V^{b}$} & $\mu$ & \multicolumn{4}{c}{$e_{v}$} \\
& 2.07 & \multicolumn{2}{c}{2.85} & 0.016 & 0.000415 & & 0.000522 \\
\hline
\end{tabular}

can abort and not the apical buds.

After this step of allocation to compartments, intra-compartment biomass allocation to each organ is computed according to specific allometric rules: bud biomass is shared between blades, petioles and internodes (it is assumed that their expansion lasts only one time step); ring biomass is spread along every axes for the diameter increment of each phytomer. Blade mass per unit area $e(n)$ varies with the $Q / D$ ratio: $e(n)=e_{\min }+e_{v} \cdot Q / D(n)$. We refer to [16] and [17] for details.

\subsubsection{Model calibration}

In September 2007, 11 individuals were felled and measured, ten at Saint-Elie Road $\left(5^{\circ} 17^{\prime} \mathrm{N}, 53^{\circ} 04\right.$ W) and one at Counami Road $\left(05^{\circ} 24 \mathrm{~N}, 53^{\circ} 11 \mathrm{~W}\right)$, in French Guiana. All the trees from Saint-Elie population were sterile and only one had branches. The tree from Counami road was pistillate and had branches.

For each tree, the target data consisted in a topological description (phytomers and axes positions) and, for each phytomer, in its internode dry mass, internode fresh length, internode fresh diameter, blade dry mass, blade (fresh) area, petiole dry mass, and infructescence dry mass. The roots were extracted and their dry weight measured for the eight youngest individuals. The ages of these trees and the delimitation of annual growth were estimated following the method presented in [12,37]: they ranged from less than 1 to 8 year-old. The measured trees also exhibit a relatively large variability in their measured characteristics: height ranged from $8 \mathrm{~cm}$ to more than $12 \mathrm{~m}$ and total aerial mass ranged from $2 \mathrm{~g}$ to around $72 \mathrm{~kg}$.

In a first step, the topology was set as observed for each tree. Specific allometries were estimated from the data to link organ dry mass to their fresh dimensions. As internode length is highly variable with the environment $[12,37]$, it was considered as an input and set directly from the measurements. The minimal value for the blade mass per unit area was $e_{\min }=0.00749 \mathrm{~g} . \mathrm{cm}^{-2}$. The ratio of underground to aerial mass was found relatively constant: 0.19 in average (SD 0.059). The sinks of blades, petioles and internodes were estimated by a linear regression of these organ masses with respect to the phytomer mass with $n=540$ data points and their values were $0.7177\left(R^{2}=0.99\right), 0.2080\left(R^{2}=0.97\right)$ and 0.07423 $\left(R^{2}=0.81\right)$ respectively.

The functional parameters of the model were estimated using the data of organ dry mass and dimensions of the 11 trees, representing in total more than 4000 data points. The results are presented in table 3.2.2. The coefficients of determination are $0.86,0.75,0.79,0.65,0.54$ for internode masses, internode diameters, blade areas, blade masses and petiole masses respectively. All trees shared the same parameter values: only the environmental factor $E^{I}$ was specific to each tree and estimated relatively to a reference value, arbitrarily chosen (equal to 10 for individual 10). The results were found consistent with visual inspection of the local environment of each tree.

\subsubsection{Alternate Patterns of Branching}

After this step of estimation of functional parameters, the values of the simulated ratio of biomass supply to potential demand at each time step can be obtained for each plant. Analyzing its variations with respect to the time of branch appearance provides a rough estimation of the threshold value: $\theta=9.975$ was found (Figure 9, for tree 10). Figure 10 shows some 3D views of the simulated trees (without infructescences). The first branch appears at time step 150, which is consistent with botanical observations. The intervals between appearance of the following successive cohorts of branches are: 48, 45, 31, 24, 45, 31, 25, 44. For 

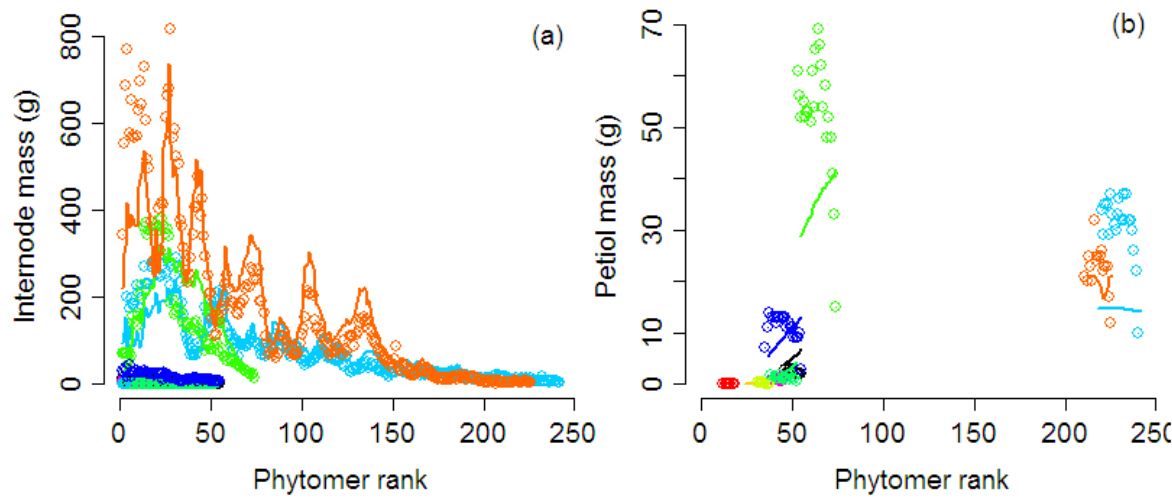

(b)
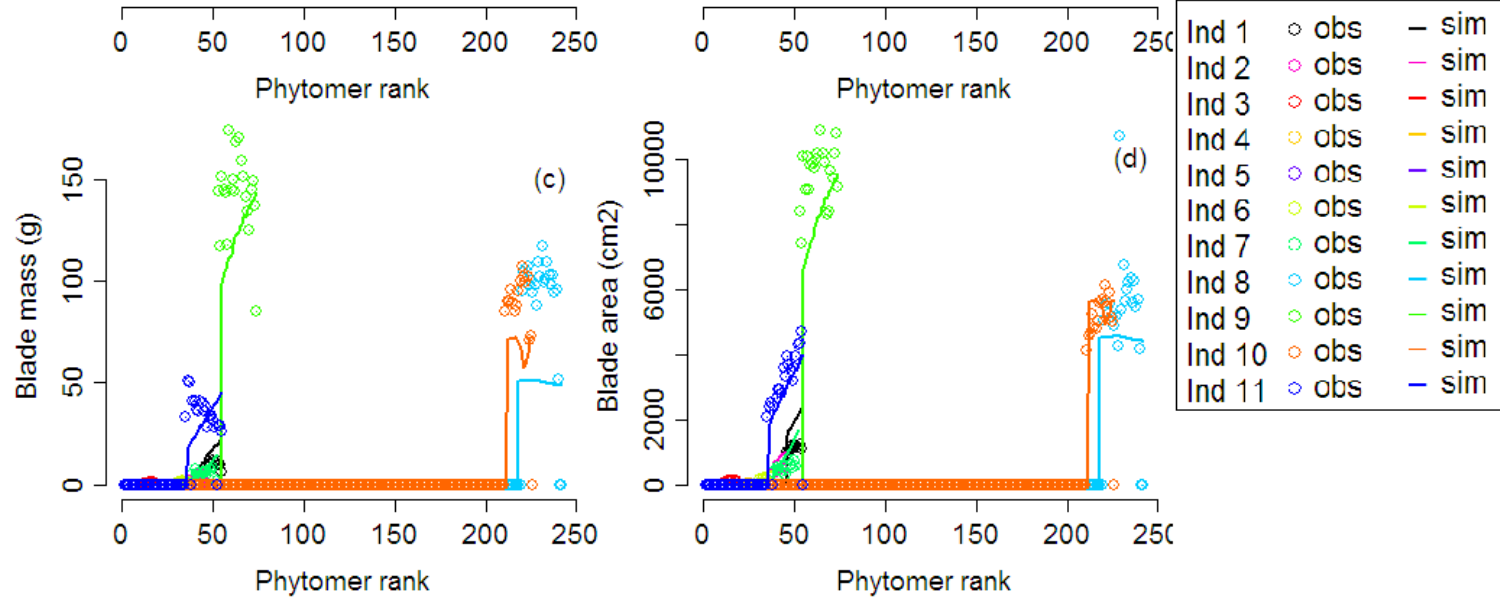

Figure 8. Graphs of simulated and observed values for internode masses (a), petiole masses (b), blade masses (c) and blade areas (d) along the main stems of the 11 individuals

C. sciadophylla, it has been observed that branches always appear at given periods of the year (once or twice a year), and since 25 phytomers are emitted each year, cohorts of branches are separated by 25 or a multiple of 25 phytomers without branches [37]. In the simulation, the number of branches per cohort regularly increases, which is unrealistic. A possible reason might be that no senescence phenomenon was included in the model, since the target trees were all young. Several alternative approaches could be considered to generate a more realistic long-term pattern: for instance, introducing the maintenance cost of organs, constraints for conduction of water and nutriments along the tree axes, or constraints for biomechanical stability.

\section{Conclusion}

The aim of this article was to present a generic dynamic model of plant growth in which the feedback control of functioning on structural development is at the origin of rhythm emergence in fructification and branching processes. Theoretical analyses were performed on two case studies concerning respectively these two phenomena. More complex versions of this model were applied to real situations on Cucumber plants and Cecropia trees. Alternating patterns were indeed observed in the measured plants. However each calibration has been made on a limited number of individuals. In particular, the threshold value for branch appearance in C. sciadophylla was estimated on an individual with one flush of branches only: this would deserve deeper investigations on other individuals with a succession of cohorts of branches. More experimental calibration should be carried out to increase the model reliability and decrease the uncertainty on parameter values.

The model is mainly based on a key variable, namely the ratio of available biomass to plant demand 


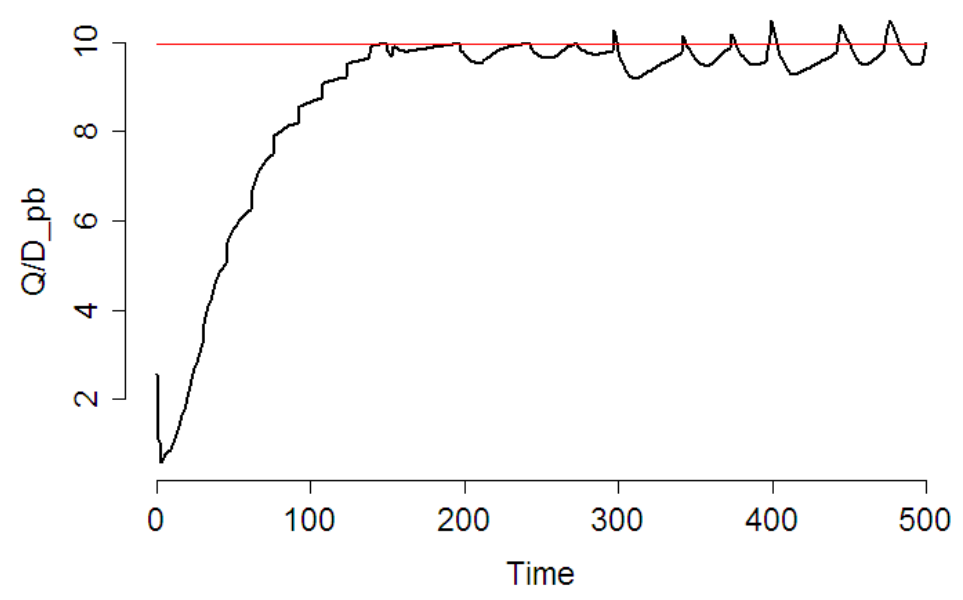

Figure 9. Variations of the Ratio of Biomass Supply to Potential Demand and Threshold for Branch Appearance
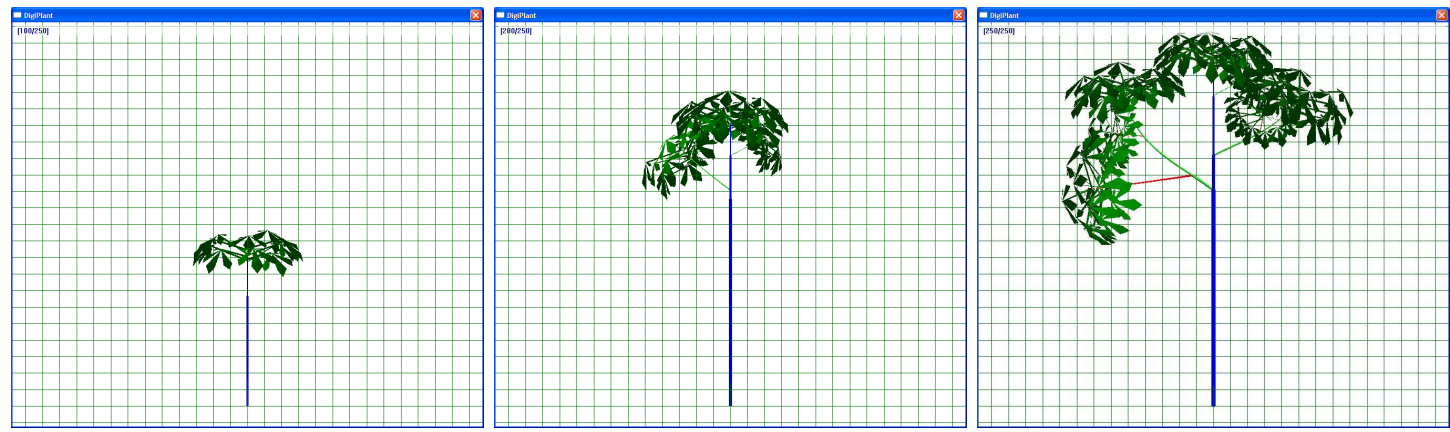

Figure 10. 3D view of Cecropia at 100, 200, and 250 time steps.

$(Q / D)$, that triggers some development events by threshold effects. In terms of model design, this seemingly simple approach can generate interesting emerging patterns. This is a satisfying strategy since it naturally introduces some feedbacks effects, thus resulting in a system with auto-regulation properties. It can therefore be successfully applied to mimick the behaviour of real plants.

However, this does not of course prove that this is the biological truth. In particular, although the tradeoff between the different growth compartment of the plant always exists, there might be other non-trophic regulations, such as hormonal control [34]. Fruit abortion or abscission is related to hormone balance, and the latter is influenced by the formation of seeds. Although the hormone theory and the assimilate theory look contrasting, Marcelis et al. [21] suggest that assimilate availability may be the trigger for a change of the hormonal balance which leads to abscission. Some other phenomena remain not considered in the model in spite of their influence on the plant growth and biomass partitioning: dominance between fruits [30], energetic cost of the structure, light effects. Regarding the branching process, our analyses suggest that the threshold on the trophic state might not be sufficient to explain the alternate branching: it has been observed that young trees with fast growth did not have branches, although their vigour state was comparable to that of older branched trees. It is hypothesized that ontogenetic factors could be involved in the regulation of branching and interact with several environmental factors: light intensity and quality, nutrient status of the soil for example. Models are developed for a better understanding of the relative effects of these different factors [6] and some of them could be integrated in the GreenLab mathematical framework. 
After the full validation of this deterministic model, a stochastic version can be implemented in order to reproduce the variability between plants that may be due to local constraints: micro-climate, pests or any other accidental damages. A simple stochastic version has been applied on pepper plants [19], where the fact that the $Q / D$ ratio exceeds a threshold is a necessary, but not sufficient, condition for fruit growth.

After a deeper validation, such a model can be a tool for virtual experiment. Pruning strategy can be simulated in the model to determine the best management practices in order to maximize the yield or get a more balanced production. Indeed, if the pruning is too heavy, the plant will not be able to grow enough fruits. But if it is too light, fruits will grow more randomly which might be less optimized.

Another perspective for this work is to pursue the mathematical study and determine the condition for rhythm generation for more complex dynamic systems. An analytical solution for the periodicity could be optimized according to the plant parameters, and this would be helpful for plant growers.

\section{Appendix: Study of the sequence with a constant demand}

The sequence we intend to study is given by equation 2.9 that we recall her:

$$
\alpha_{n+1}= \begin{cases}A \frac{1-e^{-B \sum_{d=1}^{n} d \alpha_{d}}}{\left(p_{e}+p_{a}\right)(n+1)+p^{f} \sum_{d=1}^{n+1} \delta\left(\alpha_{n+1-d}\right)} & \text { if } n<T_{e}, \\ A \frac{1-e^{T_{e}} \sum_{d=1}^{T_{n}} d \alpha_{n-T_{e}+d}}{D_{v}+p^{f} \sum_{d=1}^{T_{e}} \delta\left(\alpha_{n+1-d}\right)} & \text { if } n \geq T_{e},\end{cases}
$$

This sequence is bounded and ranges between 0 and $A=E \mu$.

While the demand remains constant (i.e no fruit or a fruit on each phytomer), the recurrence equations are:

$$
\begin{aligned}
& n<T_{e} \frac{p^{a}+p^{e}}{A}(n+1) \alpha_{n+1}=1-e^{-B n \alpha_{n}}\left(1-\frac{p^{a}+p^{e}}{E \mu} n \alpha_{n}\right) \\
& n \geq T_{e}, \frac{D}{A} \alpha_{n+1}=1-\left(1-\frac{D}{A} \alpha_{n}\right) e^{-B T_{e} \alpha_{n}} e^{B \sum_{d=n-T_{e}}^{n-1} \alpha_{d}} \\
& n \geq T_{e}, \frac{D}{A}\left(\alpha_{n+1}-\alpha_{n}\right)=\left(1-\frac{D}{A} \alpha_{n}\right)\left(1-e^{-B T_{e} \alpha_{n}} e^{B \sum_{d=n-T_{e}}^{n-1} \alpha_{d}}\right)
\end{aligned}
$$

Let note $b=B \frac{T_{e}\left(T_{e}+1\right)}{2}$ and $D=D_{v}$ if there is no fruit on the plant or $D=D_{v}+D_{f}$ if there is a fruit growing on each phytomer.

Hence, for $n \geq T_{e}$, as $\forall n, \alpha_{n}<\frac{A}{D}$, we have:

$$
\alpha_{n+1}>\alpha_{n} \Leftrightarrow \sum_{d=n-T_{e}}^{n-1} \alpha_{d}<T_{e} \alpha_{n}
$$

Proposition .1. If $\frac{A b}{D}<1$, the sequence $\left(\alpha_{n}\right)$ is decreasing.

This point can be demonstrated in a recurrent way. We note $\left(H_{n}\right)$ the hypothesis.

$$
\left(H_{n}\right): \forall d<n \alpha_{1} \leq \ldots \leq \alpha_{d} \leq \ldots \alpha_{n}
$$

We have the relationship:

$$
\frac{A b}{D}<1 \Rightarrow \forall x, \frac{A}{D}\left(1-e^{-b x}\right)<x
$$

We use it to show that 
$-\left(H_{2}\right): \alpha_{1} \leq \alpha_{2}$

Indeed, $\alpha_{2}=\frac{A}{D}\left(1-e^{-B \alpha_{1}}\right)$ and $\frac{A B}{D}<\frac{A b}{D}<1$

$-\left(H_{n}\right)$, for $n<T_{e}$

$B \sum_{d=1}^{n} d \alpha_{d} \leq B \alpha_{n} \sum_{d=1}^{n} d \leq b \alpha_{n}$, hence $\alpha_{n+1}<\frac{A}{D}\left(1-e^{-b \alpha_{n}}\right)$ and $\alpha_{n+1}<\alpha_{n}$ with the relationship .4 .

$-\left(H_{n}\right)$, for $n \geq T_{e}$

The proof is similar to the previous one.

As the sequence is positive and decreasing, it is convergent. It is possible to demonstrate in a recurrent way that $\forall n, \alpha_{n}<\beta_{n}$, where $\left(\beta_{n}\right)$ is the sequence defined by $\beta_{n+1}=f\left(\beta_{n}\right)$, with $f: x \rightarrow \frac{A}{D}\left(1-e^{-b x}\right)$. If $\frac{A b}{D}<1$ using the function $f$, we demonstrate that the sequence $\beta$ converges to 0 and so does the
sequence $\alpha$.

Acknowledgements. We are grateful to our research colleagues in agronomy and forestry who are crucial partners for this work, in both building models and providing experimental data, specifically CAU and CIRAD.

\section{References}

[1] D. Barthélémy, Y. Caraglio. Plant architecture: a dynamic, multilevel and comprehensive approach to plant form, structure and ontogeny. Annals of Botany, 99 (2007), No. 3, 375-407.

[2] C.C. Berg, P. Franco, Flora neotropica monograph 94. Cecropia. Organization for Flora Neotropica. 2005. New York Botanical Garden Press, Bronx, New York, USA.

[3] P.-H. Cournède, M.Z. Kang, A. Mathieu, J.-F. Barczi, H.P. Yan, B.G. Hu, P. de Reffye, Structural factorization of plants to compute their functional and architectural growth. Simulation, 82 (2006), No. 7, 427-438.

[4] P.-H. Cournède, V. Letort, A. Mathieu, M.Z. Kang, S. Lemaire, S. Trevezas, F. Houllier, P. de Reffye. Some Parameter Estimation Issues in Functional-Structural Plant Modelling Math. Model. Nat. Phenom, 6(2) (2011), $133-159$.

[5] J. Evers, J. Vos, X. Yin, P. Romero, P.E.L. van der Putten, P.C. Struik. Simulation of wheat growth and development based on organ-level photosynthesis and assimilate allocation Journal of Experimental Botany, 61 (2010), 2203-2216 doi:10.1093/jxb/erq025.

[6] J. Evers, A. van der Krol, J. Vos, P.C. Struik. Understanding shoot branching by modelling form and function Trends in Plant Science, 16 (2011), 464-467.

[7] T. Fourcaud, X.P. Zhang, A. Stokes, H. Lambers, C. Körner. Plant growth modelling and applications: The increasing importance of plant architecture in growth models. Annals of Botany, 101 (2008), No. 8, 1053-1063.

[8] C. Godin. Representing and encoding plant architecture: a review. Annals of Forest Science, 57 (2000),413-438.

[9] Y. Guédon, D. Barthélémy, Y. Caraglio, E. Costes. Pattern Analysis in Branching and Axillary Flowering Sequences. Journal of theoretical biology, 212 (2001),481-520.

[10] E. Heuvelink. Dry Matter Partitioning in Tomato: Validation of a Dynamic Simulation Model Annals of Botany, 77 (1996), 71-80.

[11] E. Heuvelink. Evaluation of a Dynamic Simulation Model for Tomato Crop Growth and Development Annals of Botany, 83 (1999), 413-422.

[12] P. Heuret, D. Barthélémy, Y. Guédon, X. Coulmier, J. Tancre. Synchronization of growth, branching and flowering processes in the south american tropical tree Cecropia obtusa (Cecropiaceae). American Journal of Botany, 89 (2002),No. 7,1180-1187.

[13] F. Hallé, R.A.A. Oldeman. Essai sur l'architecture et la dynamique de croissance des arbres tropicaux. Masson, Paris, 1970.

[14] S. Kirkpatrick, C. Gelatt, M. Vecchi. Optimization by simulated annealing. Science, 220 (1983), 671-680. doi: $10.1126 /$ science.220.4598.671

[15] K. Kitajima, S. Mulkey, M. Samaniego, and J. Wright. Decline of photosynthetic capacity with leaf age and position in two tropical pioneer species. American Journal of Botany, 89 (2002), No. 12, 1925-1932. Functional Plant Biology, 35 (2008), No. 10, 1243-1254.

[16] V. Letort, P. Heuret, P.C. Zalamea, E. Nicolini, P. de Reffye. Analysis of Cecropia sciadophylla Morphogenesis Based on a Sink-Source Dynamic Model International Symposium on Plant Growth Modeling and Applications (PMA09), IEEE Computer Society, Los Alamitos, CA, USA. (2009), 10-17.

[17] V. Letort, P. Heuret, P.C. Zalamea, E. Nicolini, P. de Reffye. Analysing the effects of local environment on the sourcesink balance of Cecropia sciadophylla: a methodological approach based on model inversion Annals of Forest Science, 69 (2012), 167-180. 
[18] D. Luquet, M. Dingkuhn, HK. Kim, L. Tambour, A. Clément-Vidal. EcoMeristem, a model of morphogenesis and competition among sinks in rice. 1. Concept, validation and sensitivity analysis. Functional Plant Biology, 33 (2006), 309-323. doi: 10.1071/FP05266

[19] Y.T. Ma, A.M. Wubs, A. Mathieu, E. Heuvelink, J.Y. Zhu, B.G. Hu, P.-H. Cournède, P. de Reffye. Simulation of fruitset and trophic competition and optimization of yield advantages in six Capsicum cultivars using functional-structural plant modelling Annals of Botany, 107 (2011), 793-803.

[20] L.F.M Marcelis. A Simulation Model for Dry Matter Partitioning in Cucumber Annals of Botany, 74 (1994), 43-52.

[21] L.F.M Marcelis, E. Heuvelink, L. Baan Hofman-Eijer, J. Den Bakker, L.B. Xue. Flower and fruit abortion in sweet pepper in relation to source and sink strength. Journal of Experimental Botany, 55 (2004), 22-1-2268.

[22] A. Mathieu, P.-H. Cournède, D. Barthélémy, P. de Reffye. Conditions for the Generation of Rhythms in a Discrete Dynamic System. Case of a Functional Structural Plant Growth Model 2nd International Symposium on Plant Growth Modeling, Simulation, Visualization and Applications (PMA06), IEEE Computer Society, Los Alamitos, CA, USA. (2007), 26-33.

[23] A. Mathieu, B.G. Zhang, E. Heuvelink, S.J. Liu, P.-H. Cournède, P. de Reffye. Calibration of fruit cyclic patterns in cucumber plants as a function of source-sink ratio with the Greenlab model. Proceedings of the 5th international workshop on FSPM (P. Prusinkiewicz, J. Hanan, eds.), November 2007.

[24] A. Mathieu, P.-H. Cournède, V. Letort, D. Barthélémy, P. de Reffye. A dynamic model of plant growth with interactions between development and functional mechanisms to study plant structural plasticity related to trophic competition. Annals of Botany, 103 (2009), 1173-1186.

[25] A. Mathieu, P.-H. Cournède, D. Barthélémy, P. de Reffye. Rhythms and alternating patterns in plants as emergent properties of a model of interaction between development and functioning Annals of Botany, 101 (2008), No. 8, 12331242.

[26] M.V. Mickelbart, G.S. Bender, G.W. Witney, C. Adams, M.L. Arpaia. Effects of clonal rootstocks on 'Hass' avocado yield components, alternate bearing, and nutrition. Journal of Horticultural Science and Biotechnology, 82 (2007), 460-466.

[27] S.P. Monselise, E.E. Goldschmidt. Alternate bearing in fruit trees. Horticultural Reviews. 4(1982), $128-173$.

[28] P. de Reffye, M. Goursat, J.-P. Quadrat, and B.G. Hu. The Dynamic Equations of the Tree Morphogenesis Greenlab Model. Technical Report 4877, INRIA, (2003).

[29] S. Sabatier, D. Barthélémy. Growth dynamics and morphology of annual shoots according to their architectural position in young Cedrus atlantica (Endl.) Manetti ex Carrière (pinaceae). Annals of Botany, 84 (1999), 387-392.

[30] A. Schapendonk, P. Brouwer. Fruit growth of cucumber in relation to assimilate supply and sink activity. Scientia Horticulturae 23 (1984), 21-33.

[31] J. Schupp. Alternate Bearing in Fruit Crops. PennState Technical Report, 2011: http://extension.psu.edu/fruittimes/news/2011/alternate-bearing-in-fruit-crops

[32] K. Shinozaki, K. Yoda, K. Hozumi, T. Kira. A quantitative analysis of plant form - the pipe model theory $i$. basic analysis. Japanese Journal of Ecology., 14 (1964), 97-105.

[33] R. Sievänen, E. Nikinmaa, P. Nygren, H. Ozier-Lafontaine, J. Perttunen, H. Hakula. Components of a functionalstructural tree model. Annals of Forest Sciences, 57 (2000), 399-412.

[34] U. Van Meeteren, H. Van Gelder. Role of flower buds in flower bud abscission in Hibiscus. Acta Horticulturae (1995), 284-289.

[35] J.S. Verreynne, C.J. Lovatt. The Effect of Crop Load on Budbreak Influences Return Bloom in Alternate Bearing 'Pixie' Mandarin. Journal of the American Society for Horticultural Science, 134 (2009), 299-307.

[36] J. Warren-Wilson. Ecological data on dry matter production by plants and plant communities. The collection and processing of field data (E.F. Bradley, O.T. Denmead, eds.), Interscience Publishers, New York, 1967, pp. 77-123.

[37] P.C. Zalamea, P.R. Stevenson, S. Madrinan, P.M. Aubert, P. Heuret. Growth pattern and age determination for Cecropia sciadophylla (Urticaceae). American Journal of Botany, 95 (2008), 263-271. 